\title{
Between genes and genomes - future challenges for cytogenetics
}

\author{
Ingo Schubert* \\ Department of Cytogenetics and Genome Analysis, Leibniz Institute of Plant Genetics and Crop Plant Research, Gatersleben, Germany \\ ${ }^{*}$ Correspondence: schubert@ipk-gatersleben.de
}

\section{SINCE THE VERY BEGINNING}

Cytogenetics, the science of chromosomes and chromosome complements (Sutton, 1903) originally represented the microscopic level of genetic knowledge. Chromosomes (term coined by Waldeyer, 1888) were observed during mitotic and meiotic divisions already in the second half of the 19th century. In the early days of chromosome research, when the chemical nature of chromosomes was still unclear, several remarkable observations and predictions were made. One example is the postulation that chromosomes represent genetic entities which are organized as distinct domains (territories) within the interphase nucleus (Boveri, 1909) in the same polarized orientation they occupy during anaphase (Rabl, 1885). Until the late 1960s plant (and insect) cytogenetics was a leading branch in the field, whereas human cytogenetics lagged until the establishment of tissue culture and spindle poisons to arrest metaphases. In the second half of the last century the DNA double helix was recognized as the basic structural element of a chromosome (or a chromatid after semi-conservative replication) containing genes and other sequence elements like beads on a string. In the 1980s in situ hybridization, in particular fluorescence-in situ-hybridization (FISH), was a major breakthrough in cytogenetic research and a milestone in genetics. FISH allowed the detection of specific DNA sequences on chromosomes and on interphase nuclei. "Chromosome painting" by FISH with chromosome-specific probes enabled to study interphase arrangement of individual chromosomes.

The general challenges of present-day cytogenetics are (i) to find answers to ongoing and newly arising questions by the development of novel approaches (in particular in vivo and beyond the microscopic level of resolution), and (ii) to prevent the loss of "classical" knowledge (a general risk for scientific branches with a longer tradition) on chromosome biology which was gained in earlier days of cytogenetics.

The obscure mode of chromosome compaction toward entering nuclear division is an already long-lasting challenge which remained to be solved. During this process, the chromatin is condensed by four to five orders of magnitude compared to the naked DNA double helix. Another unsolved puzzle is the reversible remodeling which chromatin domains experience during the transition from (transcriptionally) active to inactive states (or vice versa) in the course of development. Furthermore, the maintenance of centromeres over successive cell cycles, in addition to rare cases of erosion or de novo centromere formation, is an enigma which requires elucidation. In this context, it remains unclear how centromeric nucleosomes of eukaryotic chromosomes are structured from $S$ phase, when the remaining chromatin is replicated, up to the time of deposition of the centromeric histone variant $\mathrm{CENH} 3$ during G2 (or even after mitosis as in metazoans).

\section{SIZE MATTERS - BUT HOW?}

Researchers in the field of synthetic biology wish to create small novel chromosomes for scientific, breeding, or biotechnology purposes using a bottom-up approach. Other researchers try to reduce the size of natural chromosomes to the most indispensable elements (centromeres, telomeres, replication origins combined with desired sequences of interest) in a top-down approach (Houben and Schubert, 2007; Birchler et al., 2010). Both approaches have the inherent drawback; that they are based on trial and error, and thus do not provide predictable results. Moreover, we do not know why the desired small chromosomes are often much less stably transmitted through meiosis than chromosomes of "normal" size (Schubert, 2001). In this context it would be worthwhile to elucidate how birds and reptiles stably transmit chromosomes of extremely different size.

\section{SMALL IS BEAUTIFUL}

A permanent challenge for cytogenetics is to increase the resolution power of in situ hybridization and immunostaining techniques, with the goal to detect shorter nucleotide stretches or single antigen molecules reliably on chromosomes, on extended chromatin fibers and in interphase nuclei. Also the application of small but effective fluorescent chromatin tags for in vivo studies should be improved. Such improvements, together with new microscopy techniques, will be helpful to resolve the three-dimensional chromosome structure and the varying composition during interphase and nuclear divisions as well as chromosome and chromatin dynamics during mitosis and meiosis and in the context of replication, transcription, repair, or recombination processes.

A further important issue would be the precise quantification of distinct DNA double-strand breaks per nucleus, followed by tracing their possible fates, including simple ligation, sister chromatid exchange, chromosome structural aberration, deletion, or (transgene) insertion.

Gene targeting combining locus-specific endonucleases with sequence homology between trans- and target-genes, and with transgenic recombination cassettes deserves methodological development to achieve widespread application.

\section{THE FORGOTTEN MUTAGENESIS}

Another challenge is the interpretation of genomic data in the context of chromosome biology, and rules to be considered are that: (i) genomes can evolve only in accordance with chromosome structural constraints; and (ii) new terms and speculations should be avoided when existing 
knowledge can sufficiently explain the observations. An example of terms to be avoided is non-reciprocal translocation, which is neither experimentally proven so far nor needed for the explanation of genomic findings. From the 1950s till the end of the 1970s, when mutagenicity testing became an important topic for geneticists, a broad community of researchers were well trained to recognize inducible structural chromosome rearrangements during the first metaphase after their origination. Later, several tests were introduced, such as anaphase bridges, micronuclei, and the comet assay, which correlate at least with parts of metaphase chromosome aberration assays. The new tests were faster, less laborious and do not require a priori knowledge about the diversity of structural chromosome aberrations, but as a result the "first hand" experience required to identify different types of chromosome rearrangements was slowly lost. This lack of knowledge can easily lead to unnecessary misinterpretation of genomic data (Schubert and Lysak, 2011). Therefore, in the field of chromosome mutagenesis a (difficult) task for cytogeneticists is to prevent that classical knowledge is ignored.

Eventually, bridging (from either side!) the gap between molecular and the microscopic levels of our genetic knowledge will be the biggest challenge of cytogenetics for the 21 st century.

\section{ACKNOWLEDGMENTS}

I thank Jörg Fuchs, Andreas Houben, Timothy Sharbel (all IPK), Richard Jorgenson and the reviewers for critical discussions and improvements.

\section{REFERENCES}

Birchler, J.A., Krishnaswamy, L., Gaeta, R. T., Masonbrink, R. E., and Zhao, C. (2010). Engineered minichromosomes in plants. CRC Crit. Rev. Plant Sci. 29, 135-147. Boveri, T. (1909). Die Blastomerenkerne von Ascaris megalocephala und die Theorie der Chromosomenindividualität. Arch. Zellforch. 3 , 181-268.
Houben, A., and Schubert, I. (2007). Engineered plant minichromosomes: a resurrection of B chromosomes? Plant Cell 19, 2323-2327.

Rabl,C.(1885).Über Zellteilung.Morph.Jahrb. 10, 214-330.

Schubert, I. (2001). Alteration of chromosome numbers by generation of minichromosomes - is there a lower limit of chromosome size for stable segregation? Cytogenet. Cell Genet. 93, 175-181.

Schubert, I., and Lysak, M. (2011). Interpretation of karyotype evolution should consider chromosome structural constraints. Trends Genet. 27, 207-216.

Sutton, W. S. (1903). The chromosomes in heredity. Biol. Bull. 4, 231.

Waldeyer, W. (1888). Über Karyokinese und ihre Beziehungen zu den Befruchtungsvorgängen. Arch. Mikr. Anat. 32, 1-122.

Received: 03 May 2011; accepted: 30 May 2011; published online: 09 June 2011.

Citation: Schubert I (2011) Between genes and genomes - future challenges for cytogenetics. Front. Gene. 2:30. doi: 10.3389/fgene.2011.00030

This article was submitted to Frontiers in Plant Genetics and Genomics, a specialty of Frontiers in Genetics.

Copyright $@ 2011$ Schubert. This is an open-access article subject to a non-exclusivelicense between the authors and Frontiers Media SA, which permits use, distribution and reproduction in other forums, provided the original authors and source are credited and other Frontiers conditions are complied with. 